\title{
Impact of diabetes mellitus on morbidity and survival after pancreaticoduodenectomy for malignancy
}

\author{
Kunal Bikram Deo ${ }^{1,2}$, Aditya Atul Kulkarni ${ }^{1,3}$, Praveen Kumar-M ${ }^{4}$, Gautham Krishnamurthy ${ }^{1,5}$, \\ Sunil Shenvi ${ }^{1,6}$, Surinder Singh Rana ${ }^{7}$, Rakesh Kapoor ${ }^{8}$, and Rajesh Gupta ${ }^{1}$
}

\begin{abstract}
${ }^{1}$ Department of Surgical Gastroenterology, Postgraduate Institute of Medical Education and Research (PGIMER), Chandigarh, India, 'Department of Surgery, B.P. Koirala Institute of Health Sciences, Dharan, Nepal, ${ }^{3}$ Department of Surgical Gastroenterology, D Y Patil Medical College, Pune, ${ }^{4}$ Department of Pharmacology, Postgraduate Institute of Medical Education and Research (PGIMER), Chandigarh, ${ }^{5}$ Department of Surgical Gastroenterology, SRM Institutes for Medical Science, Chennai, ${ }^{6}$ Department of Liver Transplantation and Hepatobiliary Surgery, Gleneagles Global Hospital, Bangalore, ${ }^{7}$ Department of Gastroenterology, Postgraduate Institute of Medical Education and Research (PGIMER), ${ }^{8}$ Department of Radiotherapy and Oncology, Postgraduate Institute of Medical Education and Research (PGIMER), Chandigarh, India
\end{abstract}

\begin{abstract}
Backgrounds/Aims: Diabetes mellitus (DM) is a known risk factor for morbidity, length of hospital stay, or mortality after surgery, however, its impact on postoperative course and long-term survival after pancreaticoduodenectomy (PD) is not clear. Methods: This is a retrospective analysis of prospectively maintained database of 141 patients with periampullary and pancreatic head adenocarcinoma operated between January 2001 and March 2019. Clinico-pathological records and follow-up data were retrieved and analyzed. Cumulative hazard was computed for comparing the survival between DM and non-DM. Results: DM was present in 31/141 (21.9\%) patients, while 16/31 (51.6\%). were new-onset DM (NODM). Tumor size, lymphovascular \& perineural invasion, type of surgery, lymph node positivity and R0 resection rate were comparable between diabetic and non-diabetic. There was no significant difference in postoperative pancreatic fistula, delayed gastric emptying, infectious complication, hospital stay and mortality between DM and nondiabetics. Patients with DM had worse survival at 3 years (OS: HR, 3.11 [1.43-6.76] $p=0.004$, DFS: HR, 2.61 [1.23-5.53] $p=0.01$ ) and 5 years (OS: HR, 3.32 [1.46-7.53] $p=0.004$, DFS: HR, 2.87 [1.29-6.41] $p=0.009$ ). On multivariate analysis, DM (3 year OS: HR, 2.61 [1.14-5.98] $p=0.022$, DFS: HR, 2.19; $p=0.058$ ) (5 year OS: HR, 2.55; $p=0.04$, DFS: HR, $2.25 ; p=0.068$ ) and pylorus resecting surgery were significantly associated with worse survival at 3 and 5 years. Conclusions: Preoperative DM has no significant effect on postoperative course but has negative impact on 3-year and 5-year OS and DFS after PD for pancreatic and periampullary adenocarcinoma. (Ann Hepatobiliary Pancreat Surg 2021;25:230-241)
\end{abstract}

Key Words: Pancreaticoduodenectomy; Periampullary carcinoma; Pancreatic adenocarcinoma; Diabetes mellitus; Survival analysis

\section{INTRODUCTION}

Diabetes mellitus (DM) is a known risk factor for morbidity, length of hospital stay, or mortality after surgery. ${ }^{1,2}$ This is usually attributed to poor immune function, stress hormone metabolism, and disturbed vascular homeostasis due to poor glycemic control and insulin secretion. Studies have shown that patients with $\mathrm{HbAlc}$ of $\geq 6.0 \%$ and postoperative blood sugar $\geq 200 \mathrm{mg} / \mathrm{dl}$ had a higher risk (Odds ratio: 4.2, $p<0.01$ ) of developing major complications after emergency general surgery. ${ }^{3}$

Pancreaticoduodenectomy (PD) is a formidable procedure with high morbidity and mortality. ${ }^{4,5}$ The impact of DM on perioperative outcome after PD is conflicting. Earlier studies had shown that DM negatively impacts postoperative outcomes as it leads to more abdominal complications. $^{2,6}$ The complications like acute kidney injury, postoperative pancreatic fistula were found more in

Received: December 24, 2020; Revised: January 17, 2021; Accepted: February 1, 2021

Corresponding author: Rajesh Gupta

Department of Surgical Gastroenterology, Postgraduate Institute of Medical Education and Research (PGIMER), Chandigarh 160012, India Tel: +91-9216512636, Fax: +91-2744401, E-mail: rajsarakshi@gmail.com

Copyright (C) 2021 by The Korean Association of Hepato-Biliary-Pancreatic Surgery

This is an Open Access article distributed under the terms of the Creative Commons Attribution Non-Commercial License (http://creativecommons.org/ censes/by-nc/4.0) which permits unrestricted non-commercial use, distribution, and reproduction in any medium, provided the original work is properly cited Annals of Hepato-Biliary-Pancreatic Surgery • pISSN: 2508-5778 - eISSN: 2508-5859 
patients with $\mathrm{DM}^{7}$ This has been attributed to older age, increased body mass index (BMI), malnutrition, and coexisting co-morbidities in diabetic patients. However, subsequent studies showed that patients with DM did not have an increased risk of postoperative complications. ${ }^{8,9}$ Some studies have even shown the protective role of DM on postoperative pancreatic fistula (POPF). ${ }^{10}$ These authors have attributed their findings to increased deposition of pancreatic fibrosis and less pancreatic fat which makes pancreatico jejunostomy more secure with less incidence of postoperative pancreatic fistula (POPF). ${ }^{10,11}$

The effect of DM on the long-term outcome of patients undergoing PD for pancreatic and periampullary carcinoma is also debatable. Some studies have shown no effect of DM on the long-term survival. ${ }^{12,13}$ While other study shows that pancreatic cancer patients with DM enjoyed better overall survival compared to those without DM. ${ }^{14}$ On the other hand, many studies have shown poor survival in patients with DM undergoing PD for pancreatic cancer compared to non DM counterparts. ${ }^{15-17}$ This has been attributed to insulin resistance, hyperinsulinemia, and alteration of the intracellular metabolic environment which creates a favorable environment for the growth of these tumors. In addition, these subsets of patients were likely to have larger tumor with more chance of perineural invasion. We still do not have robust data on impact of DM on perioperative outcomes and survival.

In the past decade or so, the prevalence of DM has been increasing in India. ${ }^{18,19}$ Thus, it is imperative to understand impact of DM on postoperative morbidity and survival in patients who undergo such formidable procedure. We, therefore, planned the present study to look at our data to find out the role of DM in the perioperative outcome and survival in periampullary and pancreatic head adenocarcinoma undergoing pancreatoduodenectomy.

\section{MATERIALS AND METHODS}

\section{Patient selection}

Patients with periampullary and pancreatic head adenocarcinoma who underwent PD in the setting of a tertiary care referral center were included in this study. Data was retrieved from prospectively maintained records. Patients operated between January 2001 and March 2019 were included. Information related to presentation, investigations, operative and postoperative course and pathological records was retrieved. Patients with incomplete data were excluded. Follow-up data was retrieved from a prospectively maintained record of follow-up outpatient visits while additional information was received through telephonic conversation after obtaining informed consent. Patients who underwent PD for adenocarcinoma of the periampullary region and pancreatic head were included. Patients undergoing PD for non-adenocarcinoma histology were excluded. This study was approved by the Institutional Ethical Committee of PGIMER, Chandigarh (IEC/2019/ SPL-1463).

Diagnosis of periampullary and pancreatic head adenocarcinoma was established from clinical and radiological records. We included only those patients for whom histopathological confirmation of adenocarcinoma was available in the records.

We applied the diagnostic criteria of the American Diabetes Association, January 2009 for the diagnosis of preoperative DM based upon documented clinical history or laboratory studies. ${ }^{20}$ Based on duration of DM, patients were grouped as long-standing and new-onset DM (NODM). NODM was defined as diagnosis of DM within two years of diagnosis of carcinoma. ${ }^{16}$ We separately analyzed NODM and long-standing DM as subsets of DM. All patients had undergone surgery after glycemic control and clearance from endocrinologists.

Preoperative variables analyzed for the study included age, sex, clinical presentation, co-morbidities, smoking, alcohol abuse, preoperative biliary drainage like endoscopic retrograde cholangiogram (ERC) with stenting or percutaneous trans-hepatic biliary drainage (PTBD), radiological data related to location, size, relationship with vascular structures, metastasis and size of pancreatic and bile duct. Operative data included the type of surgery like PD with or without pylorus preservation, operative time and blood transfusion, tumor location, resectability, lymph nodes, pancreatic and bile duct size, and consistency of pancreatic parenchyma (firm or soft). All pancreatico-jejunal anastomosis were performed with duct-to-mucosa technique and rarely by dunking technique when duct was not identifiable.

Postoperative variables included hospital stay, morbidity (Clavien-Dindo grade) ${ }^{21}$, and need for reoperation. 
Postoperative pancreatic fistula (POPF), delayed gastric emptying (DGE), and post-pancreatectomy hemorrhage were defined as per the definition used by the Internatio- nal Study group on Pancreatic Surgery (ISGPS) ${ }^{22-24}$. All consensus and standardized criteria for definitions of complications were retrospectively applied based upon records

Table 1. Clinico-demographic and pathological comparison between DM and non DM

\begin{tabular}{|c|c|c|c|c|}
\hline Variables & DM (31) & Non DM (110) & Total (141) & $p$-value \\
\hline Age & $57.64 \pm 9.99$ & $53.21 \pm 1.15$ & $54.1 \pm 11.73$ & 0.06 \\
\hline \multicolumn{5}{|l|}{ Sex } \\
\hline Male & $18(58.1 \%)$ & $68(61.8 \%)$ & $86(61.0 \%)$ & 0.70 \\
\hline Female & $13(41.9 \%)$ & $42(38.2 \%)$ & $55(39.0 \%)$ & \\
\hline Pain abdomen & $14(45.2 \%)$ & $55(50.0 \%)$ & $69(48.9 \%)$ & 0.63 \\
\hline Jaundice & $21(67.7 \%)$ & $89(80.9 \%)$ & $110(78.0 \%)$ & 0.11 \\
\hline Weight loss & $20(64.5 \%)$ & $77(70.0 \%)$ & $97(68.8 \%)$ & 0.56 \\
\hline Alcohol & $5(16.1 \%)$ & $27(24.5 \%)$ & $32(22.7 \%)$ & 0.32 \\
\hline Smoking & $4(12.9 \%)$ & $21(19.1 \%)$ & $25(17.7 \%)$ & 0.42 \\
\hline Preoperative biliary drainage & $14(45.2 \%)$ & $57(51.8 \%)$ & $71(50.4 \%)$ & 0.51 \\
\hline \multicolumn{5}{|l|}{ Site of tumor } \\
\hline Pancreas* & $12(38.7 \%)$ & $24(21.8 \%)$ & $36(25.5 \%)$ & 0.30 \\
\hline Ampulla & $9(29.0 \%)$ & $39(35.5 \%)$ & $48(34.0 \%)$ & \\
\hline Lower third CBD & $6(19.4 \%)$ & $28(25.5 \%)$ & $34(24.1 \%)$ & \\
\hline Duodenum & $4(12.9 \%)$ & $19(17.3 \%)$ & $23(16.3 \%)$ & \\
\hline \multicolumn{5}{|l|}{ Surgery performed } \\
\hline Pylorus not preserved & $17(54.8 \%)$ & $54(49.1 \%)$ & $71(50.35 \%)$ & 0.57 \\
\hline Pylorus preserved & $14(45.2 \%)$ & $56(50.9 \%)$ & $70(49.64 \%)$ & \\
\hline Mean operative time (min) & $366.53 \pm 95.1$ & $346.57 \pm 80.18$ & $349.57 \pm 82.41$ & 0.43 \\
\hline \multicolumn{5}{|l|}{ Pancreatic consistency } \\
\hline Soft & $8(29.6 \%)$ & $33(35.9 \%)$ & $41(34.5 \%)$ & 0.55 \\
\hline Firm & $19(70.4 \%)$ & $59(64.1 \%)$ & $78(65.5 \%)$ & \\
\hline Dilated MPD & $16(66.7 \%)$ & $40(51.3 \%)$ & $56(54.9 \%)$ & 0.18 \\
\hline Vascular repair & $1(3.2 \%)$ & $4(3.6 \%)$ & $5(3.5 \%)$ & 0.19 \\
\hline Blood transfusion & $5(16.1 \%)$ & $21(19.9 \%)$ & $26(18.4 \%)$ & 0.86 \\
\hline \multicolumn{5}{|l|}{ Tumor differentiation } \\
\hline Well & $4(12.9 \%)$ & $21(19.1 \%)$ & $25(17.7 \%)$ & 0.57 \\
\hline Moderate & $21(67.7 \%)$ & $68(61.8 \%)$ & $89(63.1 \%)$ & \\
\hline Poor & 0 & $4(3.6 \%)$ & $4(2.8 \%)$ & \\
\hline NOS & $6(19.3 \%)$ & $17(15.4 \%)$ & $23(16.3 \%)$ & \\
\hline $\mathrm{T}$ stage & 28 & 99 & 127 & \\
\hline 1 & $2(7.1 \%)$ & $17(17.2 \%)$ & $19(15.0 \%)$ & 0.23 \\
\hline 2 & $10(35.7 \%)$ & $37(37.4 \%)$ & $47(37.0 \%)$ & \\
\hline 3 & $16(57.1 \%)$ & $40(40.4 \%)$ & $56(44.1 \%)$ & \\
\hline 4 & 0 & $5(5.1 \%)$ & $5(3.9 \%)$ & \\
\hline $\mathrm{N}$ stage & 29 & 95 & 124 & 0.75 \\
\hline 0 & $15(51.7 \%)$ & $57(60.0 \%)$ & $72(58.1 \%)$ & \\
\hline 1 & $12(41.4 \%)$ & $32(33.7 \%)$ & $44(35.5 \%)$ & \\
\hline 2 & $2(6.9 \%)$ & $6(6.3 \%)$ & $8(6.5 \%)$ & \\
\hline Lymphovascular invasion & $6(19.3 \%)$ & $25(22.7 \%)$ & $31(21.9 \%)$ & 0.88 \\
\hline Perineural invasion & $5(16.1 \%)$ & $21(19.0 \%)$ & $26(18.4 \%)$ & 0.75 \\
\hline Adjuvant therapy** & $15 / 28(53.6 \%)$ & $48 / 93(51.6 \%)$ & $63 / 121(52.1 \%)$ & 0.85 \\
\hline Chemotherapy only & $3(10.7 \%)$ & $9(9.7 \%)$ & $12(9.9 \%)$ & 0.87 \\
\hline Radiation only & $6(21.4 \%)$ & $18(19.4 \%)$ & $24(19.8 \%)$ & 0.81 \\
\hline Chemoradiation & $6(21.4 \%)$ & $21(22.6 \%)$ & $27(22.3 \%)$ & 0.89 \\
\hline
\end{tabular}

DM, diabetes mellitus; Non DM, non diabetes mellitus; CBD, common bile duct; MPD, main pancreatic duct; NOS, not otherwise specified *Within pancreas as site of tumor, in DM 3 out of 12 cases were periampullary carcinoma (pancreatic origin) and in non DM 5 out of 24 cases were periampullary carcinoma (pancreatic origin)

**Data of adjuvant therapy was available in 121 patients out of 141 patients. Out of these 63 patients received adjuvant therapy 
from the database.

Adjuvant therapy was given to resected cases of pancreatic head cancer based upon the patient's tolerance and performance status. Adjuvant therapy was given for tumors stage $\geq \mathrm{T} 3$, positive lymph node, positive resection margin, or presence of high-risk features like lymphovascular or perineural invasion. Choice of chemotherapy (Gemcitabine or 5-FU based regimens) \pm radiotherapy depended on the patient's profile and tolerance to toxicity. Survival analysis was done only on patients with follow-up data and in case of discrepancy telephonic conversation was made. Overall survival (OS) was defined as the time interval from the date of surgery till death or last follow-up. Disease-free survival (DFS) was defined as the time interval between the date of surgery and either date of recurrence or last follow-up without recurrence. Survival analysis was performed in patients who had at least 1-year of follow-up. While performing survival analysis by the site of the tumor, we grouped patients into pancreatic ductal adenocarcinoma and other periampullary adenocarcinoma which comprised ampullary, duodenal, and distal common bile duct adenocarcinoma.

\section{Statistical analysis}

All records of patients were maintained in the excel sheet. The analysis was performed using $\mathrm{R}$ statistical software version 3.6.1 and SPSS version 23. In $\mathrm{R}$, other than the base package ggplot $2^{25}$, pROC ${ }^{26}$, haven ${ }^{27}$, survival ${ }^{28}$, survminer $^{29}, \mathrm{ez}^{30}, \mathrm{plyl}^{31}$, were used. For the description of a continuous variable, mean \pm standard deviation was used for parametric variables, and median with interquartile range (IQR) (25th to 75th percentile) was used for nonparametric variables, and categorical variables were expressed as a column along with percentages. For inferential analysis of continuous data, parametric variables were compared using Independent t-test and non-parametric variables were compared using the Mann-Whitney $\mathrm{U}$ test and for categorical data, the comparison of two groups was done using Chi-square $\left(X^{2}\right)$ test (if all the cell in the expected contingency table is $>5$ ) and Fisher Exact test (if any one of the cells in the expected contingency table is $<5$ ). Survival analysis viz. OS and DFS were performed using a cumulative hazard in Kaplan Meier survival curves, taking patients with DM as the grouping variable, and groups were compared using log-rank $(p<0.05)$.
For assessing the effect of other variables on survival in addition to diabetes, we did Cox Regression proportional hazard model development, for which we initially assessed each variable (univariate) for its effect on 1-, 3and 5-year OS \& DFS and then based on the significance in Wald test $(p<0.05)$, we added to the multivariate cox regression model. We assessed for the global concordance of the developed model using a likelihood ratio test $(p<0.05)$ and described the hazard ratio (HR) (along with the 95\% Confidence interval (CI) for each factor both for univariate and multivariate) along with the computed $p$-value.

\section{RESULTS}

Records of 220 patients undergoing PD were available. 70 cases were excluded due to PD for diseases other than adenocarcinoma. There were 150 resected cases of adenocarcinoma of the pancreatic head and periampullary region. 9 cases of pancreatic ductal adenocarcinoma (PDAC) were excluded due to incomplete data. A total of 141 patients were analyzed for perioperative outcomes. DM was present in $31(21.9 \%)$ patients out of which NODM was present in 16/31 (51.6\%). Three patients had less than 1 year follow up and were included for analysis of the perioperative outcome. Survival data of 41 patients was incomplete and these patients were included for perioperative outcomes only. Finally, survival data of 1, 3, and 5 years was available for 97, 86, and 60 patients respectively. At 1-year, there were 23 patients with DM out of which 11 patients had NODM. At 3-year, there were 22 patients with DM and out of which 11 patients had NODM while at 5-years, there were 14 patients of DM and 6 had NODM.

Demographic characteristics and baseline variables are shown in Table 1. Pylorus preserving PD was performed in $71(50.3 \%)$ patients while pylorus was resected in 70 (49.6\%) patients. Venous resection was done in 5 (3.5\%) cases. Preoperative biliary drainage, either endoscopic or percutaneous, was performed in $71(49.3 \%)$ patients. The clinical-demographic parameters were comparable in diabetic and non-diabetic patients (Table 1). Tumor characteristics like tumor differentiation, $\mathrm{T}$ stage, $\mathrm{N}$ stage, lymphovascular invasion, perineural invasion were also similarly distributed in both groups (Table 1). There was no significant difference in the number of the patients receiv- 
Table 2. Comparison of postoperative outcomes between DM and non DM

\begin{tabular}{lcccc}
\hline \multicolumn{1}{c}{ Variables } & DM (31) & Non DM (110) & Total (141) & $p$-value \\
\hline DGE & $11(35.5 \%)$ & $35(31.8 \%)$ & $46(32.6 \%)$ & 0.70 \\
Grade A & $3(9.7 \%)$ & $15(13.6 \%)$ & $18(12.8 \%)$ & 0.71 \\
Grade B & $6(19.4 \%)$ & $13(11.8 \%)$ & $19(13.5 \%)$ & \\
Grade C & $2(6.5 \%)$ & $8(7.3 \%)$ & $10(7.1 \%)$ & \\
POPF & $9(29.0 \%)$ & $28(25.5 \%)$ & $37(26.2 \%)$ & 0.68 \\
Grade A & $4(12.9 \%)$ & $12(10.9 \%)$ & $16(11.3 \%)$ & 0.4 \\
Grade B & $5(16.1 \%)$ & $10(9.1 \%)$ & $15(10.6 \%)$ & \\
Grade C & 0 & $6(5.5 \%)$ & $6(4.3 \%)$ & 0.09 \\
Hemorrhage & $3(9.7 \%)$ & $3(2.7 \%)$ & $6(4.3 \%)$ & 0.34 \\
Surgical site infection & $9(29.0 \%)$ & $23(20.9 \%)$ & $32(22.7 \%)$ & 0.14 \\
Intraabdominal abscess & $4(12.9 \%)$ & $11(10.0 \%)$ & $15(10.6 \%)$ & 0.5 \\
Cholangitis & $1(3.2 \%)$ & $7(6.4 \%)$ & $8(5.7 \%)$ & 1.0 \\
Pneumonia & 0 & $2(1.8 \%)$ & $2(1.4 \%)$ & 0.17 \\
Bile leak & $2(6.5 \%)$ & $2(1.8 \%)$ & $4(2.8 \%)$ & 0.30 \\
Median hospital stay (range) & $13.0(6-46)$ & $11.0(6-45)$ & $11.0(6-46)$ & \\
Morbidity (Clavien-Dindo grade) & $27(87.1 \%)$ & $91(82.7 \%)$ & $118(83.7 \%)$ & 0.56 \\
$\quad<$ III & $4(12.9 \%)$ & $19(17.3 \%)$ & $23(16.3 \%)$ & $2(1.4 \%)$ \\
$\quad$ IIII & $1(3.2 \%)$ & $2(1.8 \%)$ & $3(2.12 \%)$ & 0.45 \\
Hospital mortality & $2(1.8 \%)$ & 0.63 \\
90 days mortality & & &
\end{tabular}

DM, diabetes mellitus; Non DM, non diabetes mellitus; DGE, delayed gastric emptying; POPF, postoperative pancreatic fistula

ing adjuvant chemotherapy $(55.1 \%$ vs $50.5 \% p=0.77)$ and patients receiving radiation therapy (either with chemotherapy or alone) $(41.4 \%$ vs $41.9 \% p=0.95)$ (Table 1$)$. Incidence of delayed gastric emptying (DGE), as well as postoperative pancreatic fistula (POPF), was not significantly different between the two groups (Table 2). We did not find a significant difference in complications like hemorrhage, surgical site infections, intraabdominal abscess, cholangitis, pneumonia, and length of postoperative stay (Table 2). There was one case of 90-day mortality in the diabetes group and three in the non-diabetes group, which was also not significantly different (Table 2).

We noted that patients with DM had significantly decreased OS as well as DFS at 3-year and 5-year follow-up while there was no significant difference at 1-year follow up. Subgroup survival analysis of Type 2 DM and newonset DM showed that both subgroups had significant survival hazard for OS and DFS compared to non-diabetics at 3- and 5- year while no difference was noted at 1- year survival. The cumulative hazard of all cases of DM, long-standing DM and new-onset DM at 1-, 3- and 5-year follow-up in both OS and DFS are shown in Table 2 and Fig. 1.

Univariate survival analysis of patients at 1-year fol- low-up revealed that pylorus resecting surgery, pancreatic adeno carcinoma, and positive resection margin significantly affected both OS and DFS negatively. Other factors like DM, or NODM, gender, T-stage, adjuvant therapy did not affect 1-year survival. The hazard ratio of each factor has been provided in Table 3. On multivariate analysis, only pylorus resecting surgery and pancreatic cancer had a significant negative impact on 1-year survival (Table 4). The number of each covariates and distribution of DM, NODM, and long-standing DM in pancreatic and periampullary carcinoma in survival analysis is shown in Table 5, 6 .

At 3-year follow up, factors significantly affecting survival on univariate analysis were DM, NODM, longstanding DM, pylorus resecting surgery, pancreatic adenocarcinoma, positive resection margin, and positive lymph node status (Table 3). Multivariate analysis of these factors showed that presence of DM and pylorus resecting surgery was significant factors affecting overall survival negatively at 3-year follow-up (Table 4).

Univariate survival analysis at 5-year showed that the presence of DM, NODM, long-standing DM, pylorus resecting surgery, positive resection margin, and positive lymph node status significantly affected survival (Table 3 ). 

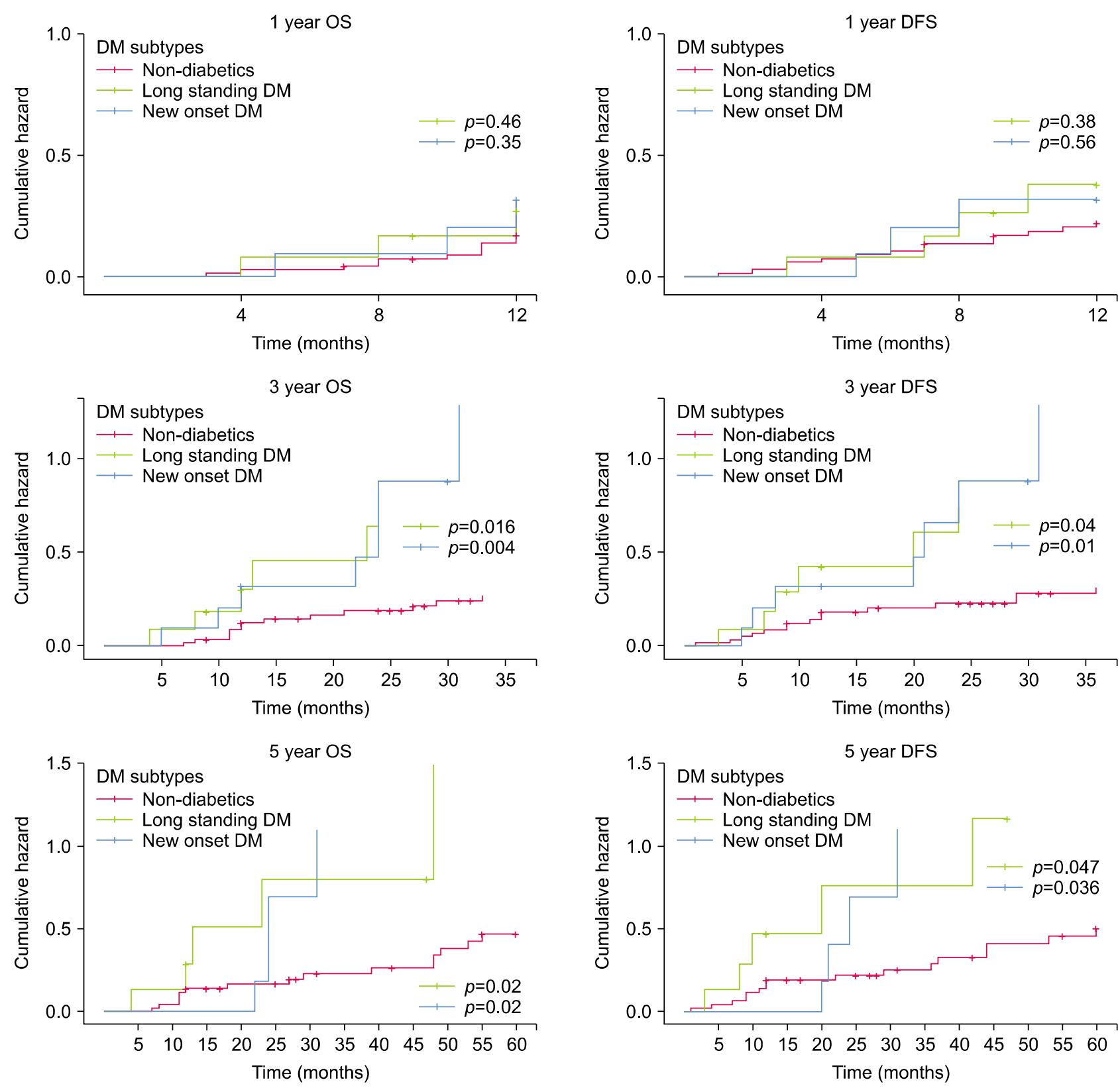

Fig. 1. Cumulative hazard plot showing 1,3, and 5-year overall survival and disease-free survival for non-diabetes, long-standing DM, and new-onset DM. $p$ values of each long standing and new onset DM are in comparison with non-diabetics. OS, overall survival; DFS, Disease free survival.

However, in multivariate analysis, DM, pylorus resecting surgery, and positive lymph node status affected survival significantly (Table 4). The global concordance of the entire developed cox-regression model was significant $(p<0.05)$ as assessed by the Likelihood test suggesting that the coefficient determined were not by chance.

\section{DISCUSSION}

Our results showed that the presence of DM did not affect postoperative morbidity, mortality or hospital stay in patients undergoing PD for periampullary and pancreatic head adenocarcinoma. On the other hand, we noted that DM had significant adverse effect on 3- and 5- year survival but not 1-year survival.

The impact of DM on morbidity and mortality after PD 


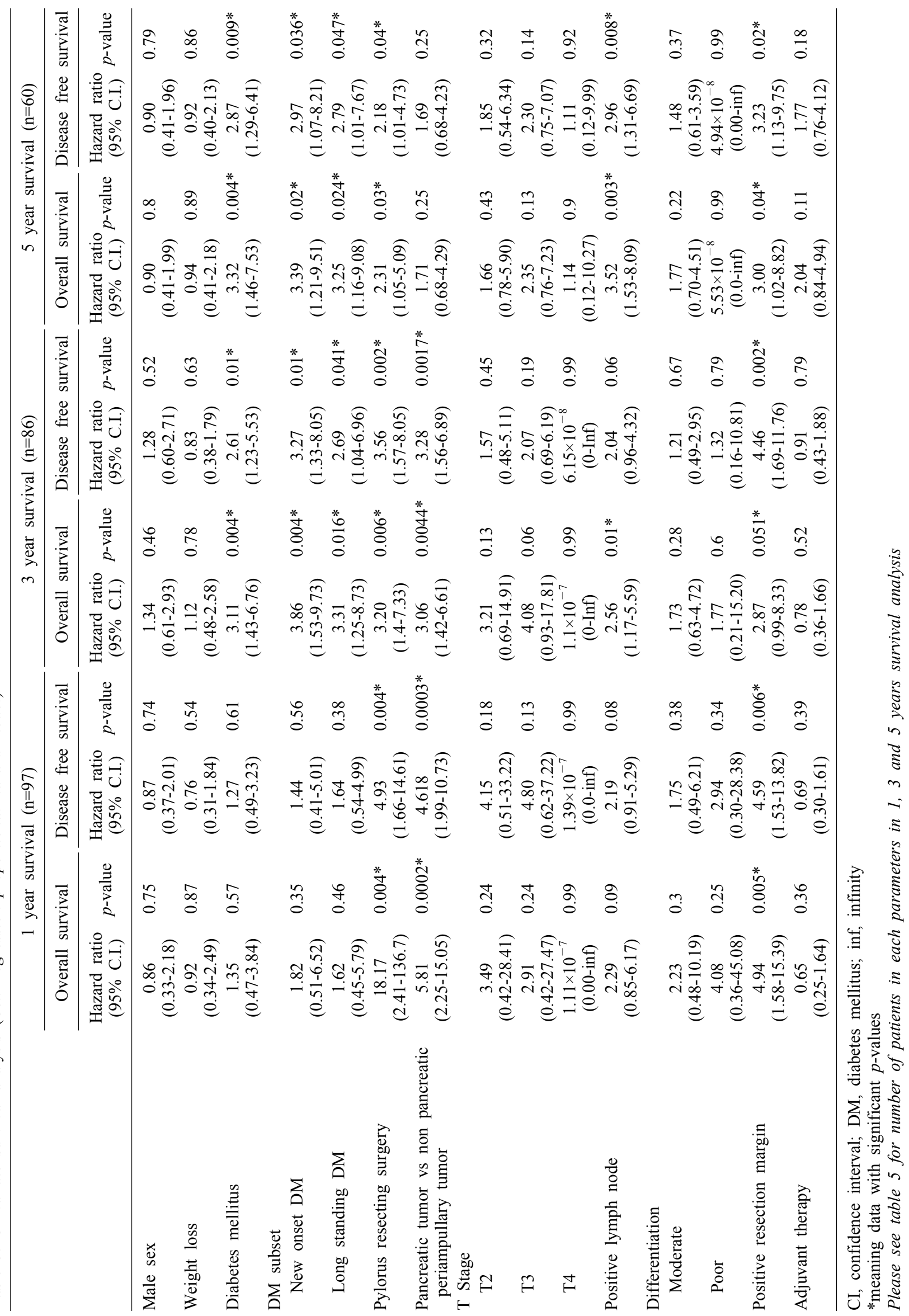


Table 4. Multivariate analysis for overall and disease free survival

\begin{tabular}{|c|c|c|c|c|}
\hline \multirow{2}{*}{ Factors } & \multicolumn{2}{|c|}{ Overall survival } & \multicolumn{2}{|c|}{ Disease free survival } \\
\hline & Hazard ratio $(95 \% \mathrm{CI})$ & $p$-value & Hazard ratio $(95 \% \mathrm{CI})$ & $p$-value \\
\hline \multicolumn{5}{|l|}{1 year survival } \\
\hline Pylorus resection & $13.51(1.76-103.78)$ & 0.012 & $4.06(1.33-12.35)$ & 0.014 \\
\hline Positive resection margin & $1.06(0.30-3.76)$ & 0.92 & $1.23(0.36-4.19)$ & 0.74 \\
\hline Pancreatic tumor & $4.78(1.55-14.76)$ & 0.006 & $4.27(1.59-11.47)$ & 0.004 \\
\hline \multicolumn{5}{|l|}{3 year survival } \\
\hline Diabetes mellitus & $2.61(1.14-5.98)$ & 0.022 & $2.19(0.97-4.94)$ & 0.058 \\
\hline Pylorus resecting surgery & $3.55(1.47-8.57)$ & 0.0047 & $3.98(1.67-9.48)$ & 0.0018 \\
\hline Positive lymph node & $2.0(0.84-4.78)$ & 0.12 & $1.42(0.61-3.31)$ & 0.41 \\
\hline Positive resection margin & $1.32(0.37-4.69)$ & 0.66 & $1.81(0.55-5.94)$ & 0.33 \\
\hline Pancreatic tumor & $1.84(0.68-4.94)$ & 0.23 & $2.10(0.78-5.67)$ & 0.14 \\
\hline \multicolumn{5}{|l|}{5 year survival } \\
\hline Diabetes mellitus & $2.55(1.04-6.27)$ & 0.04 & $2.25(0.94-5.39)$ & 0.068 \\
\hline Pylorus resecting surgery & $2.67(1.14-6.23)$ & 0.023 & $2.49(1.09-5.68)$ & 0.029 \\
\hline Positive lymph node & $3.04(1.23-7.38)$ & 0.013 & $2.46(1.02-5.93)$ & 0.044 \\
\hline Positive resection margin & $1.29(0.39-4.20)$ & 0.66 & $1.43(0.44-4.66)$ & 0.55 \\
\hline
\end{tabular}

CI, confidence interval

Diabetes mellitus (DM) here means both long standing and new onset DM combined

has been debatable. Earlier studies had shown that DM was associated with increased abdominal complication and was an independent risk factor of POPF in patients undergoing PD for pancreatic cancer. ${ }^{6,732}$ Recent study by Hank et al. ${ }^{33}$ also showed DM was associated with increased postoperative mortality after PD in pancreatic cancer. Various factors like advanced age, high BMI, malnutrition, coexisting co-morbidities were considered to be contributory. On the contrary, DeOliveira et al. ${ }^{34}$ did not find any significant association between DM and POPF, while Lin et al. ${ }^{11}$ in their retrospective study reported that DM appeared to decrease the risk of POPF. Similarly, Mathur et al. ${ }^{10}$ reported that patients with pancreatic fistula had lower incidence of DM. This finding was attributed to less pancreatic fat and increased fibrosis in pancreatic remnant. Presence of increased pancreatic fibrosis provides a firm grip to sutures, leading to secure pancreatico-jejunal anastomosis and resultant decreased POPF. Furthermore, a recent meta-analysis showed that patient with DM did not have increased overall morbidity (OR 0.90), pancreatic fistula (OR 0.88) or delayed gastric emptying (OR 1.08). The findings in the present study are also on the similar line as we also did not find impact of DM on morbidity following PD.

There is also no clarity in the literature on the impact of DM on survival after PD for periampullary and pancre- atic head adenocarcinoma. Results of our study showed that there was no significant effect of DM on 1-year survival in patients undergoing PD. Karlin et al. ${ }^{35}$ also observed no significant difference observed between DM and non-diabetics at 2-year overall survival of pancreatic cancer. A large study from UK compared 745 patients with DM in pancreatic ductal adenocarcinoma (PDAC) with 2402 PDAC without DM and found no difference in overall survival. ${ }^{36}$ However, in subset analysis, increased pancreatic cancer mortality was observed in long-standing ( $>5$ years) DM (HR 1.16). However another study showed that NODM but not long-standing DM ( $\geq 2$ years duration) was associated with reduced overall survival in patients undergoing PD for pancreatic cancer. Similarly, other clinical studies also did not find an association between DM and survival in patients with PDAC..$^{12,37,38}$ In contrast, our findings suggested that DM adversely impacted 3- and 5- year survival. The poor survival of DM after PD in pancreatic cancer has been observed in recent studies. ${ }^{15,17,33}$ The meta-analysis by Mao et al. ${ }^{15}$ showed that DM had a significantly higher risk of cancer-specific mortality and poor disease free survival (HR 1.54). Hank et al. ${ }^{33}$ showed worse median overall post resection survival in both long standing and new onset DM with pancreatic cancer. The effect was more pronounced in patients receiving neoadjuvant therapy. 
Table 5. Number of patients in each group in survival analysis

\begin{tabular}{|c|c|c|c|}
\hline \multirow{2}{*}{ Factors } & \multicolumn{3}{|c|}{ No of patients $(\%)$} \\
\hline & 1 year & 3 year & 5 year \\
\hline \multicolumn{4}{|l|}{ Sex } \\
\hline Male & $57(58.76 \%)$ & $51(59.30 \%)$ & $38(63.33 \%)$ \\
\hline Female & $40(41.24 \%)$ & $35(40.70 \%)$ & $22(36.67 \%)$ \\
\hline \multicolumn{4}{|l|}{ Significant weight loss } \\
\hline Yes & $65(67.71 \%)$ & $58(68.23 \%)$ & $41(68.33 \%)$ \\
\hline No & $31(32.29 \%)$ & $27(31.76 \%)$ & $19(31.67 \%)$ \\
\hline \multicolumn{4}{|l|}{ DM } \\
\hline Yes & $23(24.21 \%)$ & $22(26.19 \%)$ & $14(23.33 \%)$ \\
\hline No & $72(75.79 \%)$ & $62(73.81 \%)$ & $46(76.67 \%)$ \\
\hline \multicolumn{4}{|l|}{ New onset DM } \\
\hline Yes & $11(11.34 \%)$ & $11(13.09 \%)$ & $6(10.00 \%)$ \\
\hline No & $86(88.66 \%)$ & $73(86.90 \%)$ & $54(90.00 \%)$ \\
\hline \multicolumn{4}{|c|}{ Type of Pancreaticoduodenectomy } \\
\hline Pylorus resecting surgery & $45(46.39 \%)$ & $43(50.00 \%)$ & $24(40.00 \%)$ \\
\hline Pylorus preserving surgery & $52(53.61 \%)$ & $43(50.00 \%)$ & $36(60.00 \%)$ \\
\hline \multicolumn{4}{|l|}{ Site } \\
\hline Pancreas & $25(25.77 \%)$ & $21(24.42 \%)$ & $10(16.67 \%)$ \\
\hline Bile duct & $20(20.62 \%)$ & $18(20.93 \%)$ & $12(20.00 \%)$ \\
\hline Ampulla & $36(37.11 \%)$ & $32(37.21 \%)$ & $28(46.67 \%)$ \\
\hline Duodenum & $16(16.49 \%)$ & $15(17.44 \%)$ & $10(16.66 \%)$ \\
\hline \multicolumn{4}{|l|}{$\mathrm{T}$ stage } \\
\hline $\mathrm{T} 1$ & $15(16.48 \%)$ & $15(18.29 \%)$ & $14(24.14 \%)$ \\
\hline $\mathrm{T} 2$ & $34(37.36 \%)$ & $27(32.93 \%)$ & $16(27.59 \%)$ \\
\hline T 3 & $39(42.86 \%)$ & $37(45.12 \%)$ & $25(43.10 \%)$ \\
\hline T 4 & $3(3.29 \%)$ & $3(3.66 \%)$ & $3(5.17 \%)$ \\
\hline \multicolumn{4}{|l|}{$\mathrm{N}$ stage } \\
\hline $\mathrm{N} 0$ & $54(62.07 \%)$ & $47(60.26 \%)$ & $35(64.81 \%)$ \\
\hline N 1 & $30(34.48 \%)$ & $28(35.89 \%)$ & $18(33.33 \%)$ \\
\hline $\mathrm{N} 2$ & $3(3.45 \%)$ & $3(3.85 \%)$ & $1(1.85 \%)$ \\
\hline \multicolumn{4}{|l|}{ Tumor differentiation } \\
\hline Well & $22(27.50 \%)$ & $22(30.55 \%)$ & $18(33.33 \%)$ \\
\hline Moderate & $55(68.75 \%)$ & $47(65.28 \%)$ & $34(62.96 \%)$ \\
\hline Poor & $3(3.75 \%)$ & $3(4.17 \%)$ & $2(3.70 \%)$ \\
\hline \multicolumn{4}{|l|}{ Resection margin } \\
\hline Negative & $83(92.22 \%)$ & $75(92.59 \%)$ & $52(91.23 \%)$ \\
\hline Positive & $7(7.78 \%)$ & $6(7.41 \%)$ & $5(8.77 \%)$ \\
\hline \multicolumn{4}{|l|}{ Adjuvant therapy } \\
\hline Yes & $50(53.76 \%)$ & $45(53.57 \%)$ & $36(62.07 \%)$ \\
\hline No & $43(46.24 \%)$ & $39(46.43 \%)$ & $22(37.93 \%)$ \\
\hline
\end{tabular}

$\mathrm{DM}$, diabetes mellitus

Table 6. Distribution of DM and its sub types according to site of tumor in survival analysis

\begin{tabular}{lccc}
\hline \multicolumn{1}{c}{ Site of tumor } & DM & Long standing DM & New onset DM \\
\hline 1 year & 9 & $3(33.3 \%)$ & $6(66.7 \%)$ \\
Pancreas & 14 & $9(64.3 \%)$ & $5(35.7 \%)$ \\
Non pancreatic periampullary & & $2(25 \%)$ & $6(75.0 \%)$ \\
3 year & 8 & $9(64.3 \%)$ & $5(35.7 \%)$ \\
Pancreas & 14 & $1(50 \%)$ & $1(50 \%)$ \\
Non pancreatic periampullary & 2 & $7(58.3 \%)$ & $5(41.7 \%)$ \\
5 year & 12 & & \\
Pancreas & & & \\
Non pancreatic periampullary &
\end{tabular}

$\mathrm{DM}$, diabetes mellitus 
It is difficult to find out the exact mechanism behind inferior survival in diabetics with pancreatic cancer. There is increasing evidence to suggest that DM indirectly alters and reprograms the intracellular metabolic environment and makes it favorable to the proliferation of tumor cells in the pancreas. ${ }^{39,40}$ Additionally, hyperinsulinemia has been shown to enhance pancreatic cell proliferation directly or indirectly by its effect on insulin like growth factor pathway. ${ }^{39}$ Recent studies have shown that diabetic patients tend to have larger tumor, higher T-stage, increased lymph node involvement, perineural and lymphovascular invasion or advances stage in pancreatic cancer compared to non-diabetic individuals. ${ }^{33,41}$ However, findings of our study showed no difference in $\mathrm{T}$ and $\mathrm{N}$ stage between diabetic and non-diabetic groups. Interestingly, we did not find effect of DM on 1-year survival. Our results showed that tumor-related factors like site of tumor (pancreatic ductal adenocarcinoma) and surgical factors like resection margin and pylorus resection impacted early postoperative period while DM was significant factor affecting survival in patients who survived first year.

Similar to previous reports, the results of our study showed that positive resection margin, pancreatic tumor, and positive lymph node negatively affected survival. ${ }^{42,43}$ Many studies have shown that pylorus resection or preserving surgery in PD for adenocarcinoma has no significant difference on the survival. ${ }^{44,45}$ The negative impact of pylorus resection in our study could be due to selection bias because patients with large pancreatic tumors, as well as bulky lymphadenopathy are more likely to have pylorus resection, thereby, indirectly impacting survival.

The limitation of our study is that it is single center and retrospective. However, all surgeries were performed by single surgeon and that helped to eliminate technique related variabilities in the data. Besides, the survival data for $41 / 141$ (29.1\%) patients was missing. This is mainly due to patients referred for treatment to our tertiary care facility from far-off places, and they prefer to follow-up in nearby hospitals. However, in our study, the possible confounders of survival like site of a tumor i.e., pancreatic adenocarcinoma or periampullary carcinoma, type of surgery, perioperative complications, tumor stage, differentiation, nodal stage, lymphovascular and perineural invasion, and patients receiving adjuvant therapies were equally distributed in both the groups. This shows that diabetes had independent prognostic significance on 3 and 5-survival after PD for periampullary and pancreatic head adenocarcinoma. Most of the available literature is on the effect of DM on patients of pancreatic adenocarcinoma undergoing resection. Our study is one of the few studies that has looked at the effect of DM on patients undergoing PD for both pancreatic and periampullary adenocarcinoma. Results of our study show significant impact of diabetes on long-term survival even in periampullary cancers. Therefore, we believe that adverse impact of diabetes on pancreatic adenocarcinoma cannot be explained by "para-neoplastic" phenomenon alone and needs further investigations.

In conclusion, there was no significant effect of diabetes mellitus on perioperative morbidity and short term survival (1-year) after pancreaticoduodenectomy in the present study. However, presence of diabetes had significant adverse effect on 3-year and 5-year disease-free survival and overall survival in both periampullary and pancreatic adenocarcinoma undergoing resection.

\section{CONFLICT OF INTEREST}

All the authors confirm that there is no conflict of interest.

\section{SOURCE OF FUNDING}

None.

\section{ORCID}

Kunal Bikram Deo:

https://orcid.org/0000-0003-1815-6276

Aditya Atul Kulkarni:

https://orcid.org/0000-0002-3432-2484

Praveen Kumar-M:

https://orcid.org/0000-0002-9318-1167

Gautham Krishnamurthy: https://orcid.org/0000-0002-0659-5370

Sunil Shenvi: https://orcid.org/0000-0001-7708-5928

Surinder Singh Rana: https://orcid.org/0000-0001-5245-9380

Rakesh Kapoor: https://orcid.org/0000-0003-3789-1591

Rajesh Gupta: https://orcid.org/0000-0002-6585-1725 


\section{AUTHOR CONTRIBUTIONS}

Concept and plan: KBD, AAK, SSR, RG. Data on patient details and outcome: KBD, AAK, GK, SS, RK, RG. Data entry and analysis: KBD, AAK, PKM, GK, SS, RG. Manuscript preparation: KBD, AAK, PKM, RG. Manuscript finalization: KBD, AAK, SSR, RK, RG. Final approval of manuscript: KBD, AAK, RG.

\section{REFERENCES}

1. Buchleitner AM, Martínez-Alonso M, Hernández M, Solà I, Mauricio D. Perioperative glycaemic control for diabetic patients undergoing surgery. Cochrane Database Syst Rev 2012;(9): CD007315.

2. Yeh CC, Liao CC, Chang YC, Jeng LB, Yang HR, Shih CC, et al. Adverse outcomes after noncardiac surgery in patients with diabetes: a nationwide population-based retrospective cohort study. Diabetes Care 2013;36:3216-3221.

3. Jehan F, Khan M, Sakran JV, Khreiss M, O'Keeffe T, Chi A, et al. Perioperative glycemic control and postoperative complications in patients undergoing emergency general surgery: what is the role of Plasma hemoglobin A1c? J Trauma Acute Care Surg 2018;84:112-117.

4. Cameron JL, He J. Two thousand consecutive pancreaticoduodenectomies. J Am Coll Surg 2015;220:530-536.

5. Shinde RS, Pandrowala S, Navalgund S, Pai E, Bhandare MS, Chaudhari VA, et al. Centralisation of pancreatoduodenectomy in India: where do we stand? World J Surg 2020;44:2367-2376.

6. Srivastava S, Sikora SS, Pandey CM, Kumar A, Saxena R, Kapoor VK. Determinants of pancreaticoenteric anastomotic leak following pancreaticoduodenectomy. ANZ J Surg 2001;71:511-515.

7. Chu CK, Mazo AE, Sarmiento JM, Staley CA, Adsay NV, Umpierrez GE, et al. Impact of diabetes mellitus on perioperative outcomes after resection for pancreatic adenocarcinoma. J Am Coll Surg. 2010;210:463-473.

8. Kunstman JW, Healy JM, Araya DA, Salem RR. Effects of preoperative long-term glycemic control on operative outcomes following pancreaticoduodenectomy. Am J Surg 2015;209:1053-1062.

9. Nakata B, Ishikawa T, Amano R, Kimura K, Hirakawa K. Impact of preoperative diabetes mellitus on clinical outcome after pancreatectomy. Int J Surg 2013;11:757-761.

10. Mathur A, Pitt HA, Marine M, Saxena R, Schmidt CM, Howard TJ, et al. Fatty pancreas: a factor in postoperative pancreatic fistula. Ann Surg 2007;246:1058-1064.

11. Lin JW, Cameron JL, Yeo CJ, Riall TS, Lillemoe KD. Risk factors and outcomes in postpancreaticoduodenectomy pancreaticocutaneous fistula. J Gastrointest Surg 2004;8:951-959.

12. Dandona $M$, Linehan D, Hawkins $W$, Strasberg S, Gao F, Wang-Gillam A. Influence of obesity and other risk factors on survival outcomes in patients undergoing pancreaticoduodenectomy for pancreatic cancer. Pancreas 2011;40:931-937.

13. Nakai Y, Isayama $H$, Sasaki T, Mizuno S, Sasahira N, Kogure $\mathrm{H}$, et al. Clinical outcomes of chemotherapy for diabetic and nondiabetic patients with pancreatic cancer: better prognosis with statin use in diabetic patients. Pancreas 2013;42:202-208.

14. Karlin NJ, Dueck AC, Cook CB. Cancer with diabetes: prevalence, metabolic control, and survival in an academic oncology practice. Endocr Pract 2012;18:898-905.
15. Mao Y, Tao M, Jia X, Xu H, Chen $\mathrm{K}$, Tang $\mathrm{H}$, et al. Effect of diabetes mellitus on survival in patients with pancreatic cancer: a systematic review and meta-analysis. Sci Rep 2015;5: 17102.

16. Raghavan SR, Ballehaninna UK, Chamberlain RS. The impact of perioperative blood glucose levels on pancreatic cancer prognosis and surgical outcomes: an evidence-based review. Pancreas 2013;42:1210-1217.

17. Toriola AT, Stolzenberg-Solomon R, Dalidowitz L, Linehan D, Colditz G. Diabetes and pancreatic cancer survival: a prospective cohort-based study. Br J Cancer 2014;111:181-185.

18. Anjana RM, Deepa M, Pradeepa R, Mahanta J, Narain K, Das $\mathrm{HK}$, et al. Prevalence of diabetes and prediabetes in 15 states of India: results from the ICMR-INDIAB population-based cross-sectional study. Lancet Diabetes Endocrinol 2017;5:585596.

19. Ravikumar P, Bhansali A, Ravikiran M, Bhansali S, Walia R, Shanmugasundar G, et al. Prevalence and risk factors of diabetes in a community-based study in North India: the Chandigarh Urban Diabetes Study (CUDS). Diabetes Metab 2011;37:216-221.

20. American Diabetes Association. Standards of medical care in diabetes-2009. Diabetes Care 2009;32 Suppl 1:S13-S61.

21. Dindo D, Demartines N, Clavien PA. Classification of surgical complications: a new proposal with evaluation in a cohort of 6336 patients and results of a survey. Ann Surg 2004;240: 205-213.

22. Bassi C, Marchegiani G, Dervenis C, Sarr M, Abu Hilal M, Adham M, et al. The 2016 update of the International Study Group (ISGPS) definition and grading of postoperative pancreatic fistula: 11 years after. Surgery 2017;161:584-591.

23. Wente MN, Veit JA, Bassi C, Dervenis C, Fingerhut A, Gouma DJ, et al. Postpancreatectomy hemorrhage (PPH): an International Study Group of Pancreatic Surgery (ISGPS) definition. Surgery 2007;142:20-25.

24. Wente MN, Bassi C, Dervenis C, Fingerhut A, Gouma DJ, Izbicki JR, et al. Delayed gastric emptying (DGE) after pancreatic surgery: a suggested definition by the International Study Group of Pancreatic Surgery (ISGPS). Surgery 2007;142:761-768.

25. Wickham H. Ggplot2: elegant graphics for data analysis. 2nd ed. Cham: Springer, 2016.

26. Robin X, Turck N, Hainard A, Tiberti N, Lisacek F, Sanchez JC, et al. pROC: an open-source package for $\mathrm{R}$ and $\mathrm{S}+$ to analyze and compare ROC curves. BMC Bioinformatics 2011;12:77.

27. R Development Core Team. Import and Export 'SPSS', 'Stata' and 'SAS' Files (R Package Version 2.4.1). Vienna, Austria: R Foundation for Statistical Computing; 2021.

28. Therneau TM, Grambsch PM. Multiple events per subject. In: Therneau TM, Grambsch PM, ed. Modeling survival data: extending the cox model. New York: Springer, 2000:169-229.

29. R Development Core Team. Drawing Survival Curves using 'ggplot2' (R Package Version 0.3.1). Vienna, Austria: R Foundation for Statistical Computing; 2017.

30. R Development Core Team. Easy analysis and visualization of factorial experiments (R Package Version 4.4-0). Vienna, Austria: R Foundation for Statistical Computing; 2016.

31. Wickham H. The split-apply-combine strategy for data analysis. J Stat Softw 2011;40:1-29.

32. Cheng Q, Zhang B, Zhang Y, Jiang X, Zhang B, Yi B, et al. Predictive factors for complications after pancreaticoduodenectomy. J Surg Res 2007;139:22-29.

33. Hank T, Sandini M, Qadan M, Weniger M, Ciprani D, Li A, et al. Diabetes mellitus is associated with unfavorable pathologic features, increased postoperative mortality, and worse long-term survival in resected pancreatic cancer. Pancreatology 2020;20: 
125-131.

34. DeOliveira ML, Winter JM, Schafer M, Cunningham SC, Cameron JL, Yeo CJ, et al. Assessment of complications after pancreatic surgery: a novel grading system applied to 633 patients undergoing pancreaticoduodenectomy. Ann Surg 2006;244: 931-937; discussion 937-939.

35. Karlin NJ, Amin SB, Kosiorek HE, Buras MR, Verona PM, Cook CB. Survival and glycemic control outcomes among patients with coexisting pancreatic cancer and diabetes mellitus. Future Sci OA 2018;4:FSO291.

36. Hwang A, Narayan V, Yang YX. Type 2 diabetes mellitus and survival in pancreatic adenocarcinoma: a retrospective cohort study. Cancer 2013;119:404-410.

37. McWilliams RR, Matsumoto ME, Burch PA, Kim GP, Halfdanarson TR, de Andrade M, et al. Obesity adversely affects survival in pancreatic cancer patients. Cancer 2010;116:5054-5062.

38. Olson SH, Chou JF, Ludwig E, O'Reilly E, Allen PJ, Jarnagin WR, et al. Allergies, obesity, other risk factors and survival from pancreatic cancer. Int J Cancer 2010;127:2412-2419.

39. Regel I, Kong B, Raulefs S, Erkan M, Michalski CW, Hartel $\mathrm{M}$, et al. Energy metabolism and proliferation in pancreatic carcinogenesis. Langenbecks Arch Surg 2012;397:507-512.

40. Sah RP, Nagpal SJ, Mukhopadhyay D, Chari ST. New insights into pancreatic cancer-induced paraneoplastic diabetes. Nat Rev
Gastroenterol Hepatol 2013;10:423-433.

41. Hart PA, Law RJ, Frank RD, Bamlet WR, Burch PA, Petersen GM, et al. Impact of diabetes mellitus on clinical outcomes in patients undergoing surgical resection for pancreatic cancer: a retrospective, cohort study. Am J Gastroenterol 2014;109:14841492.

42. Hatzaras I, George N, Muscarella P, Melvin WS, Ellison EC, Bloomston M. Predictors of survival in periampullary cancers following pancreaticoduodenectomy. Ann Surg Oncol 2010;17: 991-997.

43. Plichta JK, Godambe AS, Fridirici Z, Yong S, Sinacore JM, Abood GJ, et al. The association between survival and the pathologic features of periampullary tumors varies over time. HPB Surg 2014;2014:890530.

44. Diener MK, Fitzmaurice C, Schwarzer G, Seiler CM, Hüttner FJ, Antes G, et al. Pylorus-preserving pancreaticoduodenectomy (pp Whipple) versus pancreaticoduodenectomy (classic Whipple) for surgical treatment of periampullary and pancreatic carcinoma. Cochrane Database Syst Rev 2014;11:CD006053.

45. Tran KT, Smeenk HG, van Eijck CH, Kazemier G, Hop WC, Greve JW, et al. Pylorus preserving pancreaticoduodenectomy versus standard Whipple procedure: a prospective, randomized, multicenter analysis of 170 patients with pancreatic and periampullary tumors. Ann Surg 2004;240:738-745. 